\title{
Bremsstrahlung Radiation At a Vacuum Bubble Wall
}

\author{
Jae-Weon Le屯* \\ School of Computational Sciences, Korea Institute for Advanced Study, \\ 207-43 Cheongnyangni 2-dong, Dongdaemun-gu, Seoul 130-722, Korea \\ Kyungsub Kim and Chul H. Lee \\ Department of Physics, Hanyang University, Seoul 133-791, Korea \\ Ji-ho Jang \\ Korea Atomic Energy Research Institute Yuseong, Daejeon 305-353, Korea
}

\begin{abstract}
When charged particles collide with a vacuum bubble, they can radiate strong electromagnetic waves due to rapid deceleration. Owing to the energy loss of the particles by this bremsstrahlung radiation, there is a non-negligible damping pressure acting on the bubble wall even when thermal equilibrium is maintained. In the non-relativistic region, this pressure is proportional to the velocity of the wall and could have influenced the bubble dynamics in the early universe.
\end{abstract}

PACS numbers: 12.15.Ji, 98.80.Cq

There have been many studies on cosmological roles of first-order phase transitions, which proceed by nucleations and collisions of vacuum bubbles [1]. For example, in electroweak baryogenesis models [2] rapid bubble expansion can provide a non-equilibrium environment, which may result in asymmetry between matter and antimatter. Furthermore, in some inflationary models [3, 4, 5], the speed of expanding vacuum bubbles determines how long the inflation period lasts. To understand the bubble kinematics in a hot plasma, it is important to study particle scatterings at a moving bubble wall. To calculate the velocity of electro-weak bubbles [6, 7, 8, 9, 10, 11] and the $\mathrm{CP}$ violating charge transport rate by the wall available for baryogenesis [2], one should know the reaction force acting on the wall due to the scattered particles, such as quarks and gauge bosons [12, 13]. (For a supersymmetric model see, for example, Ref. 14)

At the first order cosmological phase transition, the false vacuum decays to the true vacuum, which has lower energy, by making a vacuum bubble. When it is created, the wall of the bubble is at rest. As the free energy difference between the inner and the outer parts of the bubble fuels the wall, the velocity of the wall increases to the light velocity unless there is a damping force. In the literature, it is generally believed that the non-trivial damping force is caused by a deviation of the particle population from a thermal equilibrium one. In this paper, we study the effect of bremsstrahlung radiations emitted by particles on the pressure acting on a bubble wall (not necessary electroweak bubbles) during cosmological first order phase transitions.

The aim of this work is to show that, contrary to the usual arguments, the radiation damping could give a non-negligible pressure even when the particles maintain thermal equilibrium. Bremsstrahlung (braking radiation) is a radiation due to the acceleration or deceleration of a charged particle[15]. Entering a true vacuum through a bubble wall, particles interact with the wall and could acquire mass and be decelerated. For example, a fermion field $\psi$ can get mass through the well-known Yukawa term $g \bar{\psi} \phi \psi=m \bar{\psi} \psi$, where $\phi$ is a Higgs field. At this time, if the particle is charged electromagnetically, it can radiate strong electromagnetic waves due to the deceleration. Let us calculate the pressure from the scattering.

For simplicity, we assume a linear profile for the bubble wall, i.e., $g \phi(x) \equiv m(x)=m_{0} x / d$ when $0<x<d$. (See Fig.1.) and choose the coordinates of the rest frame of the bubble wall.

*Electronic address: scikid@kias.re.kr 
This approximation is good for the usual tanh profile of the wall. The radiation power of an accelerated particle is given by a relativistic version of the Larmor's formula [16]:

$$
\frac{d E_{r a d}}{d t}=\frac{A}{m^{2}}\left(\frac{d \vec{k}}{d t}\right)^{2}
$$

where $A=2 e^{2} / 3 c^{3} \simeq 0.0611$ in the natural units $(\hbar=c=k=1)$ and $\vec{k}=\left(k_{x}, k_{y}, k_{z}\right)$ is the 3-momentum of a particle. We assume a situation where this classical description of bremsstrahlung is good enough. Also, assuming that the wall is planar and parallel to the $\mathrm{y}-\mathrm{z}$ plane, we can treat the bubble as a 1 -dimensional one along the $x$-axis. The energy, momentum, and mass of the particle satisfy the usual relation

$$
E^{2} \equiv m^{2}(x)+\vec{k}^{2}(x)
$$

Let us denote the x-component of the momentum $\left(k_{x}\right)$ as $k$ from now on. Differentiating the above equation with time $t$ and using $d x / d t \equiv v$ and $k=E v$, we get the force acting on the wall due to the particles

$$
\frac{d k}{d t}=-\frac{d m^{2}}{d x} \frac{1}{2 E}
$$

which is the starting point of the pressure calculation [6]. However, if we also consider the energy carried away by the radiation $E_{\text {rad }}$, then the total energy conserved is $E_{\text {tot }} \equiv E+E_{\text {rad }}$ and the force and, hence, the pressure should be changed. From $d E_{t o t} / d t=0$, we obtain

$$
\frac{k}{E} \frac{d k}{d t}+\frac{m}{E} \frac{d m}{d t}+\frac{A}{m^{2}}\left(\frac{d k}{d t}\right)^{2}=0
$$

which has a solution for the force

$$
\frac{d k}{d t}=\frac{-m^{3}}{2 A E}\left[1-\sqrt{1-\frac{4 A E k}{m^{4}} \frac{d m}{d t}}\right] .
$$

Up to $O(A)$, one can expand the square root term and obtain

$$
\frac{d k}{d t} \simeq-\frac{d m}{d x} \frac{m}{E}-\frac{2 A}{k E}\left(\frac{d m}{d x}\right)^{2}
$$

The second term represents the radiation damping. Then, the total pressure due to the collisions of the particles in the plasma is given by $[6]$

$$
P=\int_{-\infty}^{\infty} d x \int \frac{d^{3} \vec{k}}{(2 \pi)^{3}}\left[-\frac{d k}{d t} f(E(k))\right]
$$

where $f(E)=(\exp (\beta E) \pm 1)^{-1}$ is a distribution function of fermions and bosons, respectively.

First, let us briefly review the well-known results without radiation damping. When the mean velocity of the plasma fluid $V$ relative to the wall (or the negative of the bubble wall velocity relative to the fluid ) is zero, the first term of Eq. (6) contributes

$$
\begin{aligned}
P_{1} & =\int_{-\infty}^{\infty} \frac{d m^{2}(x)}{d x} d x \int \frac{d^{3} \vec{k}}{(2 \pi)^{3}} \frac{1}{2 E} \frac{1}{e^{\beta E} \pm 1} \\
& =F\left(m_{0}, T\right)-F(0, T)
\end{aligned}
$$


where $F(\phi, T)$ is a free energy of $\phi$ at a temperature $T=\beta^{-1}$. When $V \neq 0$, the distribution function is changed to

$$
f[\gamma(E-V k)]=\left(e^{\beta \gamma(E-V k)} \pm 1\right)^{-1} .
$$

Here, $\gamma=\left(1-V^{2}\right)^{-\frac{1}{2}}$. However, using the fact that the phase factor $d^{3} \vec{k} / E$ is a Lorentz invariant and changing the integration variable to $k^{\prime}=\gamma(k-V E)$ and defining $E^{\prime} \equiv \gamma(E-V k)$, one can find that the $V$ dependency of $P_{1}$ disappears [6]. From this, it is generally believed that to get non-trivial pressure on the wall, one needs to consider a non-equilibrium deviation of $f$ [7]. Our work indicates this is not necessarily true for some phase transitions. To see this, consider the effect of the radiation (the second term of Eq. (6) ). When $V=0$, the term contributes to the pressure

$$
P_{2}=2 A \int_{-\infty}^{\infty}\left(\frac{d m(x)}{d x}\right)^{2} d x \int \frac{d^{3} k}{(2 \pi)^{3}} \frac{1}{E k\left(e^{\beta E} \pm 1\right)}
$$

which also vanishes because the second integrand is an odd function of $\mathrm{k}$. However, when $V \neq 0$, one can easily check that, due to the $1 / k$ term, the $V$ dependency survives even under the change of the integration variable. Thus, in this case,

$$
\begin{aligned}
P_{2} & =2 A \int_{-\infty}^{\infty}\left(\frac{d m(x)}{d x}\right)^{2} d x \int \frac{d^{3} \vec{k}}{(2 \pi)^{3}} \frac{1}{E k\left(e^{\beta \gamma(E-V k)} \pm 1\right)} \\
& \equiv 2 A \int_{-\infty}^{\infty}\left(\frac{d m(x)}{d x}\right)^{2} d x I_{2}(x)
\end{aligned}
$$

To be more concrete, let us calculate an approximate value of the integration when $V \ll 1$ for fermions. In this case, we can expand $f[\gamma(E-V k)] \simeq f(E)-V \beta k f(E)[f(E)-1]=$ $f(E)+V \beta k f^{2}(E) \exp (-\beta E)$. The integration of the first term gives zero, and the second term contributes

$$
I_{2}=V \beta \int \frac{d^{3} \vec{k}}{(2 \pi)^{3}} \frac{1}{E} f^{2}(E) \exp (-\beta E) \simeq \frac{(\ln 2) T V}{2 \pi^{2}}
$$

because

$$
\int \frac{d^{3} k}{(2 \pi)^{3}} \frac{1}{E} f^{2}(E) \exp (-\beta E) \simeq \frac{(\ln 2) T^{2}}{2 \pi^{2}},
$$

to lowest oder in $(m / T)^{2}$ (See Ref. 7). Therefore, for the wall described in Fig. 1 the pressure by the radiation is

$$
P_{2} \simeq \frac{(\ln 2) A m_{0}^{2} T}{\pi^{2} d} V,
$$

which is comparable to the result of numerical integration of Eq. (10) for $V \ll 1$, as shown in Fig. 2. (During the numerical study it is useful to change the measure from $d k_{y} d k_{z}$ to $2 \pi E d E$.) This pressure is proportional to the wall velocity up to the moderately relativistic case and exists even when the system is in a thermal equilibrium. During the electroweak phase transition, a particle's electromagnetic charge is not definite, so the $A$ value in Lamor's formula can not be a constant. In this paper, however, to perform a rough calculation, we have assumed that A is a constant during the phase transition. For illustration of high temperature effects on electric charges, now we consider a Debye screening of electric charge by plasma during the phase transition, which is given by effective coupling $\alpha_{\text {eff }}=\alpha /(1-2 \alpha \ln (k / \Lambda) / 3 \pi) \simeq 0.97 \alpha$, where 
we used averaged momentum $\langle k\rangle \simeq 3 T$ and $\Lambda$ of order electron mass at the last approximation (see Eq. (42) of [17]). Thus, we obtain $A=0.0599$ which is slightly smaller than the zero temperature value. We also plot the pressure with this $A$ value.

It is noteworthy that the pressure caused by the radiation damping (Eq. (13)) is of order $O(\alpha)$, which is bigger than the pressure due to a departure from thermal equilibriums $7,18,19$ ] $\left(O\left(\alpha^{2}\right)\right)$ [18], and hence non-negligible. Here, $\alpha$ is the fine structure constant. Note also that the power of bremsstrahlung due to bubble walls is much stronger $(O(\alpha))$ than that of ordinary bremsstrahlung of electrons colliding with ions in a plasma $\left(O\left(\alpha^{3}\right)\right)$ 20]. Since the electroweak phase transition is a complicated phenomenon, by no means is our work a full calculation of the pressure acting on the electroweak bubbles. The purpose of this paper is to present a general idea that radiation damping (although usually ignored in the many related works for bubble wall velocity calculations) could give rise to significant frictional forces even in thermal equilibrium states at some cosmological phase transitions. To include the effects of other particles (e.g., gluon and W/Z particles) in our work, we need to modify Larmor's formula by using some sort of group factor. Even in this case, it is hardly probable that the pressure from the radiation damping from different gauge sectors exactly cancel each other. Hence, one can expect that a $O(\alpha)$ viscosity to survive. Since bubbles are slow initially, they are supposed to be in a thermal equilibrium state initially. An ordinary calculation shows no friction at this time, but the radiation damping force exists in this stage, and hence, this pressure can significantly change the early evolution of the vacuum bubbles, and the nature of electroweak baryogenesis or inflationary cosmology.

\section{ACKNOWLEDGEMENTS}

The authors are thankful to Myongtak Choi for helpful discussions. This work was supported in part by the Korean Science and Engineering Foundation and Korea Research Foundation (BSRI-98-2441).

[1] C. H. Lee, J. Korean Phys. Soc. 33, 588 (1998).

[2] M. Trodden, Rev. Mod. Phys. 71, 1463 (1999).

[3] D. La and P. J. Steinhardt, Phys. Rev. Lett. 62, 376 (1989).

[4] D. S. Goldwirth and H. W. Zaglauer, Phys. Rev. Lett. 67, 3639 (1991).

[5] S. Koh, J. Korean Phys. Soc. 49, 787 (2005).

[6] N. Turok, Phys. Rev. Lett. 68, 1803 (1992).

[7] G. Moore and T. Prokopec, Phys. Rev. Lett. 75, 777 (1995).

[8] P. J. Steinhardt, Phys. Rev. D 25, 2074 (1982).

[9] K. Enqvist, J. Ignatius, K. Kajantie, and K. Rummukainen, Phys. Rev. D 45, 3415 (1992).

[10] M. Dine, R. G. Leigh, P. Huet, A. Linde, and D. Linde, Phys. Rev. D 46, 550 (1992).

[11] C. H. Lee, J. Korean Phys. Soc. 32, 861 (1998).

[12] D. B. K. Andrew G. Cohen and A. E. Nelson, Nuc. Phys. B 349, 727 (1991).

[13] G. R. Farrar and M. E. Shaposhnikov, Phys. Rev. D 50, 774 (1994).

[14] P. John and M. G. Schmidt, Nucl. Phys. B 598, 291 (2001).

[15] K. T. Byun, K. Y. Kim, and H. Y. Kwak, J. Korean Phys. Soc. 47, 1010 (2005).

[16] J. Jackson, Classical Electrodynamics, 2nd ed. (Wiley, New York, 1975).

[17] R. A. Schneider, Phys. Rev. D66, 036003 (2002).

[18] G. D. Moore, JHEP 0003, 006 (2000).

[19] G. D. Moore and T. Prokopec, Phys. Rev. D 52, 7182 (1995).

[20] S. Ichimaru, Basic Principles of Plasma Physics (W. A. Benjamin, Reading, MA., 1973). 


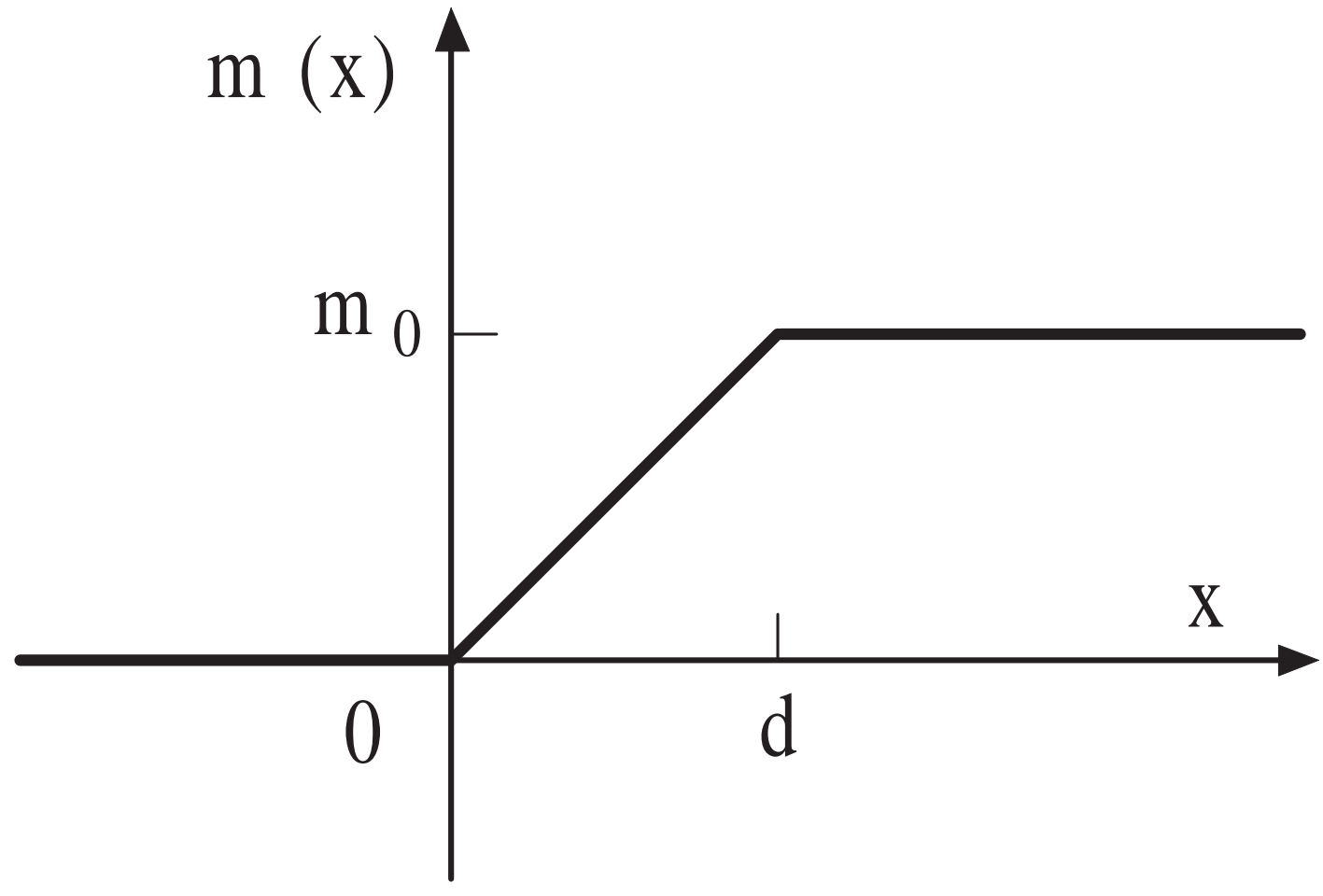

FIG. 1: The effective mass of the particle $m(x)$ in the wall rest frame. 


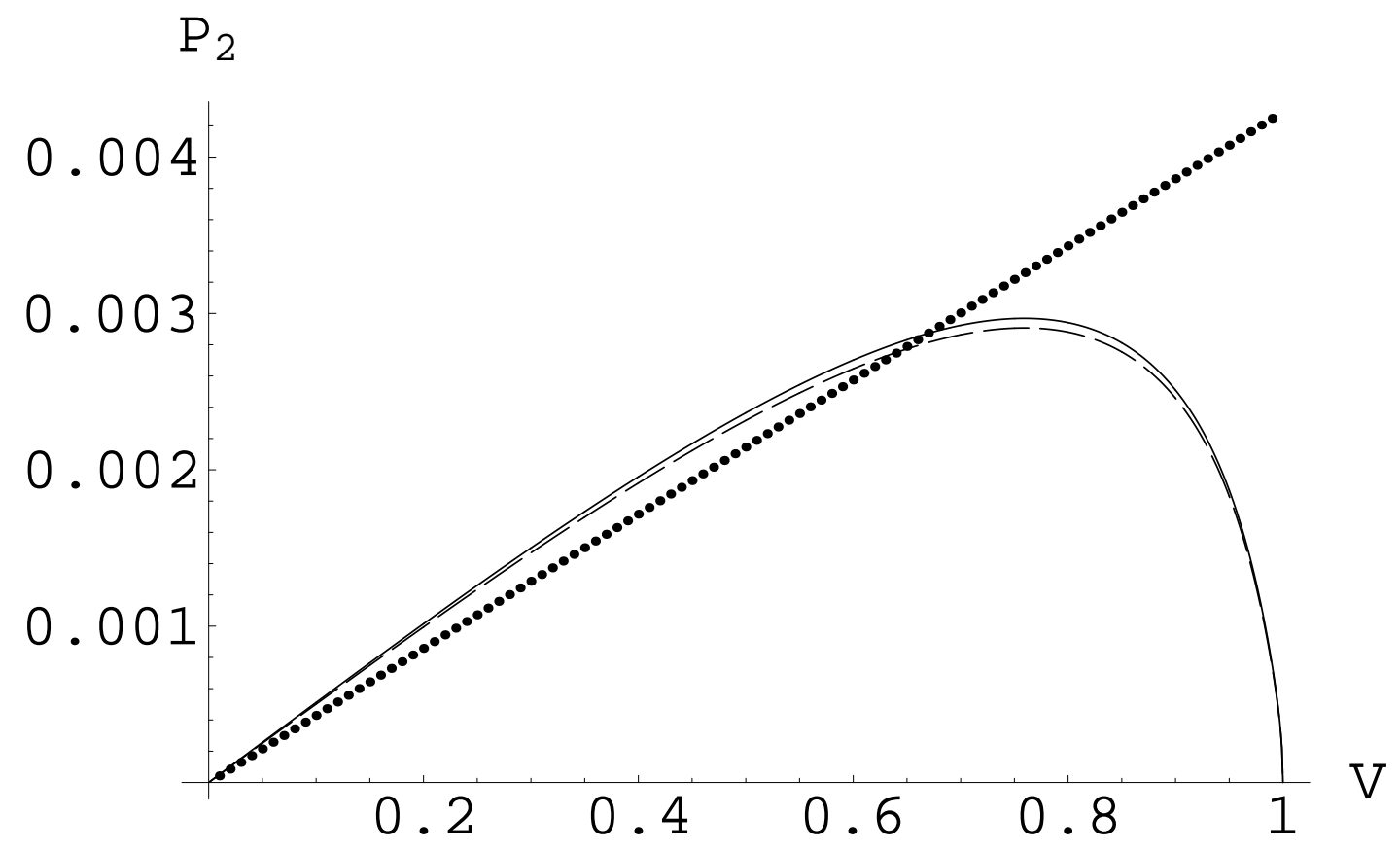

FIG. 2: The pressure by the radiation damping of fermions colliding with the linear bubble wall as a function of the wall velocity. The thick line shows numerical integration of Eq. (10) and the dotted line shows the approximate formula in Eq. (13). Here we set $1 / d=1=m_{0}=T$ for simplicity. The dashed line represents the result with Debye screening of charge. 